\title{
Canadian Association of Gastroenterology policy on the application for, and implementation of, clinical practice guidelines
}

\author{
Harminder Singh $\mathrm{MD}^{1}$, Grigorios I Leontiadis $\mathrm{MD} \mathrm{PhD}^{2}$, Lawrence Hookey $\mathrm{MD}^{3}$, Robert Enns MD ${ }^{4}$, \\ Lana Bistritz MD , Louis-Charles Rioux MD ${ }^{6}$, Louise Hope ${ }^{7}$, Paul Sinclair MSc ${ }^{7}$
}

H Singh, GI Leontiadis, L Hookey, et al. Canadian Association of Gastroenterology policy on the application for, and implementation of, clinical practice guidelines. Can J Gastroenterol Hepatol 2014;28(9):473-480.

An important mandate of the Canadian Association of Gastroenterology (CAG), as documented in the Association's governance policies, is to optimize the care of patients with digestive disorders. Clinical practice guidelines are one means of achieving this goal. The benefits of timely, high-quality and evidenced-based recommendations include:

- Enhancing the professional development of clinical members through education and dissemination of synthesized clinical research;

- Improving patient care provided by members by providing focus on quality and evidence;

- Creating legislative environments that favour effective clinical practice;

- Enhancing the clinical care provided to patients with digestive disease by nongastroenterologists; and

- Identifying areas that require further information or research to improve clinical care.

The present document provides the foundation required to ensure that clinical practice guidelines produced by the CAG are necessary, appropriate, credible and applicable. These recommendations should be adhered to as closely as possible to obtain CAG endorsement.

\section{La politique de l'Association canadienne de gastroentérologie sur les propositions et la mise en œuvre des directives cliniques}

Comme le démontrent les politiques de gouvernance de l'Association canadienne de gastroentérologie (ACG), l'un des mandats important de l'association consiste à optimiser les soins aux patients ayant des troubles digestifs. Les directives cliniques font partie des moyens pour réaliser cet objectif. Des recommandations de qualité, opportunes et fondées sur des données probantes comportent les avantages suivants :

- Améliorer le perfectionnement professionnel des membres cliniciens par la formation et la diffusion de la synthèse des recherches cliniques.

- Améliorer les soins aux patients fournis par les membres en s'attardant sur la qualité et les données probantes.

- Créer des environnements législatifs qui favorisent des pratiques cliniques efficaces.

- Accroître les soins cliniques des non-gastroentérologues aux patients ayant des troubles digestifs.

- Déterminer les secteurs où il faut obtenir plus d'information ou effectuer plus de recherches pour améliorer les soins cliniques.

Le présent document fournit les assises nécessaires pour s'assurer que les directives cliniques produites par l'ACG soient utiles, pertinentes, crédibles et applicables. Il faut adhérer le plus étroitement possible aux recommandations pour obtenir l'aval de l'ACG.

Key Words: Clinical Practice Guidelines, Methodology, GRADE

\section{THE PROCESS FOR CLINICAL PRACTICE GUIDELINE DEVELOPMENT}

Clinical practice guidelines (CPGs) are defined as systematically developed recommendations with associated background summary of potential benefits and harms and quality of evidence to assist practitioner and patient decisions about appropriate health care for specific clinical circumstances. CPGs should offer recommendations about the usefulness of screening strategies, specific diagnostic tests and/or treatments for a given disorder. The goal of the CPG is to optimize clinical practice, and includes avoiding unnecessary or inaccurate diagnostic tests as well as ineffective or harmful treatments. Therefore, CPGs should be particularly considered for areas with existing wide variations in clinical practice. The steps involved in the development and dissemination of CPGs, endorsed or developed by the Canadian Association of Gastroenterology (CAG), are discussed in the following sections.

\section{Selecting a topic}

a. The need for a particular CPGs will be determined by one, or more, of the following criteria:

i. needs assessments from survey of the membership;

ii. proposals brought forward by one or more active CAG members; and

iii. topics identified as priorities by the CAG Board and Clinical Affairs, particularly in topics for which new, important evidence has emerged.

b. CPGs will be considered if there is literature to support an evidence-based approach and if there is a clear clinical need.

c. Proposals will be made in writing to the VP-Clinical Affairs (see the Checklist for CPG Proposals; Appendix I). Proposals will be reviewed semiannually by Clinical Affairs. Final approval of a CPG proposal will be made by the CAG Board. A scoping review

${ }^{1}$ Past Lead CAG Practice Affairs, University of Manitoba, Wininpeg, Manitoba; ${ }^{2}$ Lead CAG Practice Affairs, McMaster University, Hamilton, Ontario;

${ }^{3}$ VP CAG Clinical Affairs, Queen's University, Kingston; ${ }^{4}$ Past VP CAG Clinical Affairs, University of British Columbia, Vancouver, British

Columbia; ${ }^{5}$ VP CAG Administrative Affairs, University of Alberta, Edmonton, Alberta; ${ }^{6}$ Lead CAG Ethics Committee, University of Montreal,

Montreal, Quebec; ${ }^{7}$ Canadian Association of Gastroenterology, Oakville, Ontario

Correspondence: Mr Paul Sinclair, Executive Director, Canadian Association of Gastroenterology, 224, 1540 Cornwall Road Oakville, Ontario L6J 7W5. Telephone 905-829-2504 / 888-780-0007, e-mail cagoffice@cag-acg.org

Received and accepted for publication August 13, 2014 
of the literature may be required before submission of a CPG proposal to determine whether the evidentiary base is sufficient to support the development of a CPG. If a CPG already exists in a given area, justification for an update initiative must be included in the proposal (particularly if the existing CPG is a CAGsponsored initiative).

d. All aspects of CAG-approved CPG initiative must be administered via the CAG National Office, including funding. If the CPG is being developed in partnership with another organization, then clear lines of accountability and financial oversight must be provided with the proposal. Where possible, external sources of funding should be sought, such as the Canadian Institutes of Health Research (CIHR), Canadian Partnership Against Cancer (CPAC), etc.

\section{Selecting the CAG Steering Committee and the CPG Panel}

a. Upon Board approval of a CPG, CAG Clinical Affairs will select one to two individuals to serve as Chair/Co-chairs of the CPG steering committee. The Chair/Co-chairs ideally will not be involved in the original submission of the CPG application to the CAG. CAG Clinical Affairs may also make recommendations for membership of the Steering Committee.

b. The CPG Steering Committee Chair/Co-chairs will be responsible for the selection of members who have both/either clinical interests and content expertise in the topic at hand.

c. CPG Panel membership: If the CPG is deemed to require the meeting of a CPG Panel, membership of the panel will include CAG members but may also require representation from other fields (eg, surgery, family practice, international experts). Representation from other medical disciplines, nursing and patient advocates should be considered. Selection of the Canadian members on the CPG Panel should also consider variables such as regional distribution, sex, pediatric expertise, ethical expertise, and academic versus community-based practice. Although inclusion of at least one CPG methodology expert is recommended, it is also essential all CPG Panel members become familiar with the current methodology and grading system (Grading of Recommendations Assessment, Development and Evaluation [GRADE]; see below) used by the CAG, before the start of the CPG Panel deliberations.

d. Before finalization of panel membership, each individual must complete a conflict of interest declaration statement. These statements will be reviewed by the Ethics Chair/Advisory subcommittee (see section 9; Framework for the management of conflict of interest).

e. In certain situations, joint CPGs with other specialty societies may be advantageous, both nationally and internationally. The CAG would work in partnership with other organizations to appoint members to a CPG development panel when collaboration with these organizations to develop joint recommendations is deemed beneficial. For each collaborative guideline panel, the CAG will develop an a priori agreement with partner organizations on how key guideline elements (such as conflict of interest and disagreement on final recommendations) will be handled in the event of disagreement between the organizations.

3. Identifying key questions and scope of the CPG recommendations

a. Key questions should focus on critical outcomes (benefits and harms) important to patients and could reflect areas of controversy or uncertainty that will benefit from a systematic evidence-based review.

b. CPG Panel members are urged to limit the scope of the initiative to issues that can be resolved with available clinical literature, questions that are relevant to practitioners of gastrointestinal medicine, and topics that can be addressed within the time and energy constraints of the CPG process. Authors should also note that long CPG documents, and those with excessive numbers of recommendations, can be difficult to publish in peer-reviewed journals and may have limited impact.

c. The CPG Panel should also focus on, and define, the following factors to produce a focused, coherent and readable document:

i. which patient or practice settings will be examined? (eg, inclusion and exclusion criteria);

ii. which aspects of care will be addressed? (eg, screening, therapy, diagnosis, prognosis);

iii. what outcomes will be influenced by the CPG? (eg, morbidity, mortality, quality of life, organizational outcomes, economics); and

iv. who are the target users of the CPG?

Steering Committee members should, in their planning, follow one of the proposed methodologies outlined in the CMA Handbook on Clinical Practice Guidelines (1), Canadian Task Force on Preventive Health Care Methods for CPG development and GRADE assessment (2), and/or "Guidelines 2.0: Systematic development of a comprehensive checklist for a successful guideline enterprise" (3).

\section{Systematic literature review}

a. The purpose of the systematic review of the literature is to determine whether:

i. $\quad$ PG(s) on a particular topic already exist; and

ii. medical evidence exists that will impact on the formulation of the CPG.

When possible, it may be preferable to adapt an existing CPG on a particular topic rather than to develop one de novo. Retrieved guidelines should be assessed for their quality using a recognized guideline appraisal system such as the AGREE (www.agreetrust.org) instrument. Guidelines of sufficient quality can then be assessed for their acceptability and applicability to the topic at hand. Some, or all, of the specific recommendations in a particular guideline could be adapted by the working group. The ADAPTE (www.g-i-n.net/document-store/working-groups-documents/ adaptation/adapte-resource-toolkit-guideline-adaptation-2-0.pdf/ view?searchterm=adapte) Collaboration provides a useful and validated framework to guide this process.

b. If a decision is made to proceed with a systematic review, it is recommended that the systematic review be carried out with the aid of a medical information specialist. The CAG has engaged the Upper GI Cochrane group (McMaster University, Hamilton, Ontario) to perform systematic searches for CAG CPGs (Appendix II and III) although this is not mandatory provided an alternative approach will provide high-quality output.

Past reviews, meta-analyses and previous guidelines may be considered to summarize historical data. Literature searches should cover at least 10 years, unless representing an update on a previoius CPG. Literature addressing economics and/or quality of care should also be included where available. In general, medical evidence should be restricted to peer-reviewed sources. Data presented in abstract form only should, as a rule, not be considered. However pivotal studies in abstract form could be included provided that the source is designated as non-peerreviewed and deemed critical for an adequate appraisal of the topic. Three good resources for learning more about how to perform a systematic review include: http://www.shef.ac.uk/scharr/ sections/heds/sys_rev; http://www.york.ac.uk/inst/crd/index.htm; and http://www.cochrane.org.

Guidelines, like primary studies, may be retrieved using systematic search techniques, but because some (eg, NICE guidelines) are only published by organizations and not in journals, other search strategies may be required. The National Guidelines Clearinghouse (www.guidelines.gov) is considered to be the most comprehensive website on which to find guidelines on a given topic. 
TABLE 1

\title{
Grading of Recommendations Assessment, Development and Evaluation (GRADE) rating of evidence and strength of recommendations for therapeutic interventions*
}

\author{
Quality of evidence $^{\dagger}$ \\ High Further research is very unlikely to change confidence in the estimate of effect \\ Moderate $\quad$ Further research is likely to have an important impact on the confidence in the estimate of effect and may change the estimate \\ Low Further research is very likely to have an important impact on the confidence in the estimate of effect and is likely to change the estimate \\ Very low Any estimate of effect is very uncertain \\ Strength of recommendations ${ }^{\ddagger}$
}

Strong Desirable effects of an intervention outweigh its undesirable effects (strong recommendation for an intervention) or the undesirable effects of an intervention outweigh its desirable effects (strong recommendation against an intervention). A strong recommendation implies that most people will be best served by the recommended course of action. Adherence to such recommendations can be readily used as a performance indicator to judge the quality of the clinical care

Weak

Weak recommendations are those for which the desirable effects probably outweigh the undesirable effects (weak recommendation for an intervention) or undesirable effects probably outweigh the desirable effects (weak recommendation against an intervention) but appreciable uncertainty exists. A weak recommendation implies that the guideline developers believe most individuals would be best served by the recommended course of action, but some would not be. For clinicians, the implication is that they must recognize that different choices are appropriate and they must help each person arrive at a management decision consistent with their own values and preferences, ie, shared decision making is important. Policy-making will require substantial debate and involvement of various stakeholders. Weak recommendations result when the balance between desirable and undesirable effects is small, the quality of evidence is lower, and there is more variability in the values and preferences of patients.

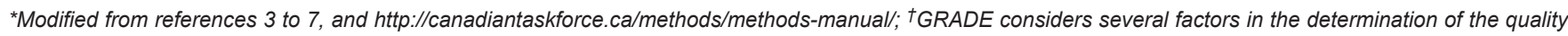
of the evidence. As a starting point, evidence from randomized controlled studies begins as high quality, while evidence from observational studies begins as low quality evidence. Evidence can then be downgraded depending on several factors: evidence is downgraded based on consideration of risk of bias, inconsistency, indirectness, imprecision and publication bias. Evidence is upgraded based on large effect, dose response, and if all possible confounding and biases would have reduced the demonstrated effect; $\neq$ For determining the strength of recommendations, the quality of evidence, the balance between the benefits and harms, the values and preferences of patients, and the resource implications of an intervention should be considered. The quality of evidence and the balance between the benefits and harms are the most important considerations. Strong recommendations are made when there is a large difference between the benefits and harms and certainty around that difference, greater certainty or similarity in patient values and preferences and the quality of the evidence is higher. Weak recommendations are made when there is some uncertainty
\end{abstract}

c. It is critical that the GRADE methodology is followed to develop the CPGs and rate the:

i. quality of the evidence; and

ii. strength of the recommendation(s).

Eighty percent or more of the CPG Panel members should agree with the grading of the evidence and recommendations. A summary description of GRADE for therapeutic interventions is shown in Table 1.

All CPG Panel members are expected to be familiar with the basic concepts of GRADE methodology as described in recent publications from the GRADE working group (eg, the 2008 BMJ series [4-9]) and the GRADE website http://www.gradeworkinggroup.org/index.htm).

Note that CPGs that include recommendations on diagnostic tests or diagnostic strategies should also follow the GRADE methodology, but present unique challenges (6). A summary description of GRADE for diagnostic tests/strategies is shown in Table 2.

\section{Development of recommendations}

a. The CPG Panel typically will generate a set of recommendations. It is anticipated that the wording of each recommendation will require multiple iterative revisions until consensus among the panel is achieved, following a modified Delphi process, similar to that used in previous CAG CPGs (10-12). If a formal consensus conference is planned, it is suggested that the proposed recommendations be circulated to the entire CPG Panel for at least two rounds of iterative revisions before the consensus conference.

b. Recommendations should be worded to provide clear and unambiguous guidance to the practitioner. This can be achieved by specifying the patients to whom the recommendation applies, the intervention, and the outcome expected to change. Active verbs such as "do", "offer", "give" and "counsel to" provide clearer guidance than passive verbs such as "should be considered". If there is uncertainty regarding the robustness of the statement, it should be reflected in the strength of the recommendation grading. Authors should avoid using obtuse and/or evasive language in an attempt to improve apparent statement grading at the cost of making the recommendations less guiding.

c. Where evidence exists, recommendations should include reference to the pediatric population.

d. Each individual recommendation will be accompanied by a grading matrix that will include:

i. grading of the quality of medical evidence; and

ii. grading of the strength of the recommendation.

e. Face-to-face meetings are preferred when feasible. However, communication by e-mail, teleconference and videoconference may also be appropriate leading to a final consensus conference.

f. In most cases, it is advisable that a formal consensus process (such as a modified Delphi technique) be adopted by the CPG Panel.

g. Where consensus has been obtained, those members rejecting the statement or accepting with major reservations, should be allowed to voice their dissenting position and have it recorded in the meeting minutes for insertion in the final manuscript.

h. Voting should be reported in an anonymous aggregate fashion using technology such as electronic touch pads or online voting.

i. Where possible, an impartial facilitator should be used to guide the discussion at a consensus conference (if required). The facilitator and Chair/Co-chairs should be nonvoting positions.

j. Each initiative should include a discussion about how the CPG will be updated in the future to maintain its clinical relevance.

6. Reporting

a. A final draft of the CPG must be prepared in a timely fashion following the conclusion of the consensus process. Ideally, this should be accomplished within six months.

b. The Steering Committee is responsible for the content of the final draft. All CPG Panel members should be given ample opportunity for input into the final wording of the report.

c. The final draft of the report will be posted on a members only section of the CAG website for a period of two weeks for 
TABLE 2

Grading of Recommendations Assessment, Development and Evaluation (GRADE) for diagnostic tests/strategies; differences from GRADE for therapeutic interventions*

\section{Factors that can influence and decrease quality of evidence Differences from quality of evidence for therapeutic interventions}

Study design $\quad$ - When randomized controlled trials or observational studies have compared two or more diagnostic tests or strategies and have reported their effect on patient-important clinical outcomes (such as mortality, morbidity, or quality of life), then the GRADE approach is similar to the classic approach for therapeutic interventions. However, such studies are yet uncommon.

- To date, most of the diagnostic studies compare two or more diagnostic test/ strategies and only report diagnostic accuracy outcomes (such as sensitivity and specificity). Such outcomes are surrogates for patient-important clinical outcomes; the CPG Panel has to make inferences about the likely impact of the use of a diagnostic test/strategy on patient-important clinical outcomes. Evidence from cross sectional or cohort studies that report diagnostic accuracy starts as high quality evidence. However, such studies are very vulnerable to high risk of bias and indirectness of outcomes, and typically end providing low quality evidence.

Risk of bias A valid tool for assessment of the risk of bias for diagnostic accuracy studies should be used, such as the QUADAS-II tool (Ann Intern Med 2011;155:529).

Indirectness Criteria similar to GRADE for therapeutic interventions

Inconsistency Criteria similar to GRADE for therapeutic interventions

Imprecision Criteria similar to GRADE for therapeutic interventions

Publication bias Criteria similar to GRADE for therapeutic interventions

*Modified from reference 6. Note, for determining the strength of recommendations for diagnostic tests/strategies, the CPG panel should consider the same criteria as for the recommendations for therapeutic interventions; that is, the quality of evidence, the balance between the benefits (reported patient-important clinical outcomes or presumed patient-important clinical outcomes resulting from the accuracy of the test) and harms (complications of the test), the values and preferences of patients, and the resource implications of an intervention

membership review and input (note: only feedback that identifies grave errors, or is factual and supported by published evidence will be considered by the Steering Committee).

d. In generating the CPG report, the developers should consider developing tools that will enhance the use of the report (eg, decision aids, algorithms, patient and physician educational material and practice tools)

e. It is suggested that the report writers adhere to the check-list developed by the Conference on Guideline Standardization (http://gem.med.yale.edu/cogs/) or incorporated in the AGREE tool. The purpose of this statement is to define a standard for guideline reporting that will promote guideline quality and facilitate implementation.

f. Developers should consider publication of CAG-supported CPGs in the Canadian Journal of Gastroenterology and Hepatology.

\section{Dissemination:}

a. The CPG report must be submitted to a peer-review process with the intent of publication.

b. Request for dissemination will not be entertained from parties who are not sponsors of the CPG initiative.

c. The CAG will simultaneously contact all sponsors of a CPG initiative to advise on publication and to gauge interest in further dissemination initiatives. The CAG must be involved in any dissemination projects of the CPG (beyond original publication), in whole or in part, which include any reference to the CAG.

d. Interested parties should contact the CAG directly with a proposal, inclusive of project details, time lines and budget.

e. Other potential tools for dissemination include:

i. posting on the CAG website and e-Portal;

ii. making the CPG available to the National Guideline Clearinghouse;

iii. presentation at Canadian Digestive Diseases Week and regional meetings; and

iv. other initiatives such as collaboration with other specialty societies, presentations at national/international meetings and research trials should also be considered.

\section{Funding}

The CAG is not in a financial position to continuously fund the development and update of multiple CPGs on its own. If industry support is required, multisponsorship is advantageous and will be sought out, ideally in a "blind trust" fashion. Only in extraordinary circumstances will single-industry sponsorship be allowed with prior approval from the CAG board. Funding sources for any CPG will be disclosed during the process and in any publication arising as a result of process. Funding sources will not influence the CPG process in any way. All funding obtained for a particular CPG will be handled as a "grant-inkind" through the CAG office. In situations where there is an excess of funds, these will be either applied to the publication costs and dissemination of the CPG or toward the development of other CPGs identified as a priority. CPG organizers are strongly encouraged to seek funding from federal and provincial granting bodies that offer programs to support consensus endeavors (eg, CIHR, CPAC). Funding of CPG Panel members will be in keeping with CAG guidelines for visiting speakers (economy airfare, modest setting and hotels).

9. Framework for the management of conflict of interest

Preamble: Collaboration among physicians, medical researchers and pharmaceutical/biotechnology companies can enhance patient care by promoting the discovery and development of new treatments. However, relationships between individual physicians and the industry may create conflicts of interest, potentially resulting in undue influence on professional judgment, particularly in the CPG development process $(13,14)$.

Because CPG reports are intended to guide current standards of care, it is essential that potential conflicts of interest (COI) be handled in a consistent fashion. The purpose of this framework is to outline steps required to identify and manage potential COIs.

Ethics and conference funding

To minimize the risk of COI, bias or undue influence, funding for any CAG-sponsored CPG will adhere to the following principles:

a. no direct industry funding of participants;

b. underwriting of the CPG through unrestricted, pooled industry funds from multiple sponsors where possible; and

c. financial transparency (see section 8). 


\section{The COI management process}

A. Identification of COI

1. Each proposed member of the CPG Panel must provide full disclosure of all potential sources of $\mathrm{COI}$ by completing and submitting a declaration of COI form for the 24-month period before the start date of the consensus process (Appendix IV). Disclosures will pertain to proposed members, their spouses and close family members.

2. Potential competing interests will include both financial and nonfinancial interests.

Financial interests with industry could include:

- Declared research funding or support

- $\quad$ Stock (investments)

- Honoraria

- Consultant

- Speakers bureau

- Employment

- $\quad$ Corporate Board positions

Nonfinancial interests could include:

- Intellectual property (patents, copyrights)

- Prior provision of public positions/statement/expert testimony

- Employee/contractor relationships with government agencies, health ministries, cancer boards, third-party drug benefit plans, government lobby groups, public advocacy agencies and foundations

\section{B. Assessment of disclosures}

1. The CPG Steering Committee will decide on the most appropriate option for $\mathrm{COI}$ assessment and management from the following options:

a. All CPG participants disclose their potential COI in writing, and these disclosures are reviewed by the CAG Ethics Committee Lead

b. A formal COI subcommittee may be formed to guide assessment and management of COI.

i. Chair: The COI subcommittee will be chaired by the CAG Ethics Committee Lead.

ii. Membership: Committee membership will include the CPG Steering Committee Chair/Co-Chairs, VP-Clinical Affairs, and the CAG Executive Director (ex officio).

iii. Mandate and tasks: The CAG Ethics Committee Lead will advise on the identification and management of $\mathrm{COI}$ issues involving CPG Panel members and will report as needed to the other members of COI advisory subcommittee.

iv. Role of the Chair: Review, clarify and advise on the precise role(s) of industry sponsors, if any:

o review, identify and rank items likely to implicate commercial or financial consequences;

o review, comment and advise on the aggregate results of the COI disclosure forms, to understand the nature and extent of the declared conflicts of CPG Panel members; and

o identify and advise on other likely 'hot spots' for the CPG process.

v. Process:

o the COI subcommittee will review participants' disclosures and classify them as minimal, moderate or significant $\mathrm{COI}$; and o launching of the CPG initiative will be conditional on COI committee approval of the proposed membership. This process should occur within a reasonable time frame (20 days) so as to not delay the CPG development unduly.

2. Review of the disclosure statements will be carried out by one of the processes outlined above, before the finalization of the CPG Panel members.

a. Each member's COI declaration statement will be classified as:

i. minimal COI (advisory board participation, speakers honoraria, member of public advocacy group or foundation);

ii. moderate COI (consultant, speaker's bureau); or

iii. significant COI (research funding, stockholder, employee of pharmaceutical firm, holder of intellectual property, government or governmental agency employee or contractor).

3. Members of the CPG Panel may report concerns regarding potential COI of another member in writing to the CAG Ethics Committee Lead for reassessment.

4. Disclosure information for each member, and the process of assessment and management of actual or potential COI, must be part of the final CPG report and related publications.

\section{Framework for managing declared COI}

Management of declared COIs must balance the need for unbiased opinion and discussion with the potential loss of valuable or critical information through the exclusion of content experts who have disclosed an actual or potential COI. As such, a COI should not necessarily preclude participation in a $\mathrm{CPG}$ provided that the $\mathrm{COI}$ framework is adhered to.

1. The Chair of COI subcommittee or CAG Ethics Committee Lead may consider excluding from the CPG Panel any member who is believed to have a real COI that may seriously impact the actual or perceived integrity and validity of the consensus process. This will be assessed on a case-by-case basis, and justified in writing to the CPG Steering Committee and VP-Clinical Affairs.

2. The group consultation process of the $\mathrm{CPG}$ will be considered to be in camera. Industry partners will not be present at the consensus conference or in any part of the process.

3. The options for management of a declared COI are:

a. A priori stratification of each participant according to their declared significant $\mathrm{COI}$ and its relevance to a particular issue

b. If a member of the panel is believed to have a significant COI that could impact the actual or perceived integrity and validity of the consensus process, this COI will be reported by the CAG Ethics Committee Lead or COI Subcommittee Chair, as applicable. The member will be contacted by the chair of COI subcommittee to review the possibility of their stepping down from the discussion and voting process.

c. Otherwise, for any participant who would present minimal or moderate COI, a reporting approach by which all participants are permitted to discuss and vote but the degree of conflict for each voter is reported in the manuscript will be followed. 


\section{Singh et al}

\section{APPENDIX 1}

\section{Checklist for a clinical practice guideline (CPG) proposal to the Canadian Association of Gastroenterology (CAG)}

$\square$ We have provided the rationale that supports the need for this (needs assessment, scientific advancement, changing clinical/epidemiological parameters)

$\square$ Yes, there is literature available to support an evidence-based approach

$\square$ We have provided a proposed timeline for development of this CPG and listed the anticipated output and publication(s)

$\square$ We request that the CAG administrate this initiative; otherwise, we have provided a rationale for this initiative to be administered beyond the CAG

$\square$ We understand that the CAG will source multisponsorship for this initiative. Where sponsorship is already available, or interest has been indicated, we have provided a list of the sponsor(s) and their commitment

$\square$ We agree that ALL funding related to this initiative will be administered by the CAG, or where funds will not be administered by the CAG, we have providedjustification for this

$\square$ We have provided a list of proposed CPG panel members and Chair, listing the rationale for each candidate (CAG committee representative, regional representation, expertise, etc).

$\square$ We understand that the CAG will appoint a Chair (who will not be one of the organizers) for this CPG after discussion with the CPG organizers and we have provided a list of proposed individuals for this role.

$\square$ We agree with the process outlined by the CAG Policy on the Application for, and Implementation of, Clinical Practice Guidelines. Any modification of theseguidelines, with respect to this initiative, has been explained thoroughly.

$\square$ We agree to abide by the ethical principles and requirements, as outlined in these guidelines

\section{APPENDIX II}

\section{Search process for guidelines}

The following details the steps to be taken for the drafting and delivery of literature searches. The sequential steps will take, at minimum, three days to complete entirely, with a realistic expectation of five to seven days to complete without delays in communication.

Dividing the scope of the We will draft a single, large literature search to identify all potentially relevant papers to the entire guideline. If there are greater than review to manageable 5000 citations from all the databases, we will subdivide the search strategy into several smaller strategies such as screening, diagnosis, proportions treatment, etc. Alternatively, the search could be divided into up to 10 specific questions (eg, barium for dysphagia diagnosis)

Determining what needs to be searched

We will liaise with a selected person from the guideline development team to determine what specific terms should be included in the search. For example, you may decide it is unnecessary to search definitions if you have already established that a standard one will be used (eg, Rome III for functional dyspepsia)

Defining the type of evidence to be sought

The iterative nature of the search process The guideline development group will provide guidance on the type of papers to be sought (ie, primary studies, editorials, letters)

The search strategy will be drafted and the terms agreed with the contact person from the guideline development team. This part of the search process usually involves testing terms to examine their effects on the search results. Several communications between the searcher and guideline contact person will likely be required

Confirming resources to be We will run literature searches on Medline, EMBASE and the Cochrane Register of Controlled Clinical trials databases. We would searched not normally search the grey literature. The guideline development group should inform us whether additional databases need to be searched

Delivery of search results We will edit the search results to remove duplicate citations, and any in vitro or vivo (animal) studies. We will download the subject headings associated with each citation for inclusion in the Reference Manager database to facilitate searching. The results of the literature searches will be collated and e-mailed to the guideline group as either a Word document or a text file compatible with a Bibliographic Management software format (eg, Reference Manager, Endnote) for importing, or both

Selecting papers We will select and include only studies relevant to the guideline topic. If requested, results will be divided into subgroups (eg, studies of adults, children and mixed groups)

It is the responsibility of the guideline development group to add the appropriate paper to the appropriate statement within the guideline

Document delivery The guideline group is responsible for obtaining copies of papers. However, we are happy to attempt to obtain any papers that may be difficult to find 


\section{APPENDIX III}

\section{Search strategy formulation}

The following details the steps to be taken for the drafting and delivery of literature searches to support the development of guidelines

Drafting a concise strategy begins with formulating a precise question. EBM convention recommends following the "PICO(S)" format. This includes identifying all synonyms for terms related to your specific:

1. Patient population

2. Intervention(s)

3. Comparison intervention(s)

4. Outcomes

5. Setting

Searching a simple question, a search string would combine each of the above groups with the "AND" operator. Depending on the nature of the question, it may be necessary to use the "OR" operator to combine intervention(s) with comparison intervention(s). The Centre for Evidence Based Medicine (UK)* offers good guidance on asking focused questions and formulating search strategies

Consider using both text words and controlled vocabulary to ensure the strategy is as thorough as possible. Text words appear in the titles and/or abstracts of a publication. Controlled vocabulary or subject headings are pre-defined terms that are applied by the database indexers to describe the topics mentioned in the record. Include all the ways your relevant concepts can be expressed (i.e. truncation, adjacency operators, variation in spelling).

The "NOT" operator is then applied to exclude particular results. This is commonly done to exclude solely animal studies from search results. In EMBASE (through OVID) this is done combining the search results with "NOT (animal\$ not human\$).sh,hw."

If necessary, further limits can be applied to the search results to restrict to age of subjects, gender, publication type and language of publication.

*Asking Focused Questions. Available from <http://www.cebm.net/index.aspx?o=1036> Accessed June 17, 2012

\section{APPENDIX IV}

\section{CONFLICT OF INTEREST DISCLOSURE FORM FOR} PARTICIPANTS \& SPEAKERS IN CAG ACCREDITED CLINICAL PRACTICE GUIDELINE DEVELOPMENT

\section{Objective:}

The purpose of this document is to identify real, potential and perceived conflicts of interest that may impact on the integrity of the CPG development process.

\section{Definitions:}

1. A real conflict of interest occurs when a participant in the CPG process has outside interests or connections (industry, government, granting agencies, third party payers) that influence the ability to act with integrity, objectivity, and independence.

2. A potential conflict of interest may arise when a participant in the CPG process has a private or personal interest, such as an identified future commitment, with an involved party

3. A perceived conflict of interest arises when a reasonably well-informed person has a justifiable belief that a participant has a conflict of interest, even if there is no conflict.

\section{Disclosure:}

You must take reasonable steps to disclose to CAG all financial and fiduciary relationships, which may represent real, potential or perceived conflicts of interest over the past 24 months. This declaration applies to yourself, your spouse and close family members. The COI Advisory Subcommittee will review this document and advise on the management of any COI according to the Management Framework for CAG sponsored CPG's 


\section{CONFLICT OF INTEREST DISCLOSURE FORM FOR PARTICIPANTS \& SPEAKERS IN CAG ACCREDITED CLINICAL PRACTICE GUIDELINE DEVELOPMENT}

Your Name:

Date:

CPG Event:

The Canadian Association of Gastroenterology must ensure balance, independence, objectivity, and scientific rigor in all business, educational and scientific activities. Accordingly, you are expected to disclose any potential, apparent or actual interests or connections (business, personal, professional, volunteer or other) appear to influence your ability to act with integrity, objectivity, and independence towards the assigned task. The intent of this initiative is to provide CAG and interested parties with information on the interests or relationships that could influence interpretations, recommendations, and conclusions.

Over the past 24 months, have you or your spouse received financial support from a commercial entity for any clinical or research activity connected to the CPG development process? Do you have any other financial relationship with a commercial entity, which might be perceived as having a connection to this CPG?

NO, I do not have any relevant financial relationships with any commercial interests. I agree to immediately notify the CAG National Office if a significant financial interest develops at any time prior to or during my participation in the above educational event, presentation or task

YES If yes, please complete the table below listing: Please include additional pages, as required.

\begin{tabular}{|l|l|l|l|}
\hline \multirow{2}{*}{ Company or Organization* } & \multicolumn{3}{|c|}{ Nature of relevant financial relationship } \\
\cline { 2 - 4 } & What was received?+ & For what role?\# & \\
\hline & & & \\
\hline & & & \\
\hline & & & \\
\hline & & & \\
\hline & & & \\
\hline
\end{tabular}

* A company or organization could be a pharmaceutical firm; a third party drug benefits provider or a governmental agency + Honoraria, consultants fees, stock holdings, contractual agreements, research grants.

\# Membership on speaker's bureaus or ad boards, consultant, employee or stockholder.

By signing below, I declare that the above is true to the best of my current knowledge and understanding

Signature:

Date:

\section{REFERENCES}

1. Connor Gorber S, Singh H, Pottie K, Jaramillo A. Process for guideline development by the reconstituted Canadian Task Force on Preventive Health Care. CMAJ 2012;184:1575-81.

2. Canadian Task Force on Preventive Health Care. Methods Manual $<$ http://canadiantaskforce.ca/methods/methods-manual/ and GRADE Manual http://canadiantaskforce.ca/methods/grade/> (Accessed June 2014).

3. Schünemann HJ, Wiercioch W, Etxeandia I, et al. Guidelines 2.0: Systematic development of a comprehensive checklist for a successful guideline enterprise. CMAJ 2014;186:E123-42.

4. Guyatt GH, Oxman AD, Vist G, et al; for the GRADE Working Group. Rating quality of evidence and strength of recommendations GRADE: An emerging consensus on rating quality of evidence and strength of recommendations. BMJ 2008;336:924-6.

5. Guyatt GH, Oxman AD, Kunz R, Vist GE, Falck-Ytter Y, Schünemann HJ; GRADE Working Group. Rating quality of evidence and strength of recommendations: What is "quality of evidence" and why is it important to clinicians? BMJ 2008;336:995-8.

6. Schünemann HJ, Oxman AD, Brozek J, et al; for the GRADE Working Group. Grading quality of evidence and strength of recommendations for diagnostic tests and strategies. BMJ 2008;336:1106-10.

7. Guyatt GH, Oxman AD, Kunz R, et al; for the GRADE Working Group. Rating quality of evidence and strength of recommendations: Incorporating considerations of resources use into grading recommendations. BMJ 2008;3361170-3.
8. Guyatt GH, Oxman AD, Kunz R, et al; for the GRADE Working Group. Rating quality of evidence and strength of recommendations: Going from evidence to recommendations. BMJ 2008;336:1049-51.

9. Jaeschke R, Guyatt GH, Dellinger P, et al; for the GRADE Working Group. Grading quality of evidence and strength of recommendations for diagnostic tests and strategies. BMJ 2008;336:1106-10.

10. Sadowski DC, Bernstein CN, Bitton A, Croitoru K, Fedorak RN, Griffiths A; for the CAG Crohn's Consensus Group. Canadian Association of Gastroenterology Clinical Practice Guidelines: The use of tumor necrosis factor-alpha antagonist therapy in Crohn's disease. Can J Gastroenterol 2009;23:187-202.

11. Barkun A, Bardou M, Marshall JK; for the Nonvariceal Upper GI Bleeding Consensus Conference Group. Consensus recommendations for managing patients with nonvariceal upper gastrointestinal bleeding. Ann Intern Med 2003;139:843-57.

12. Armstrong D, Marshall JK, Chiba N, et al. Canadian Consensus Conference on the management of gastroesophageal reflux disease in adults - Update 2004. Can J Gastroenterol 2005;19:15-35.

13. Canadian Medical Association. Code of Ethics. (update 2004); $<$ www.cma.ca/En/Pages/code-of-ethics.aspx > (Accessed September 11, 2014).

14. Clinical practice guidelines and conflict of interest. CMAJ 2005; 173:1297. 


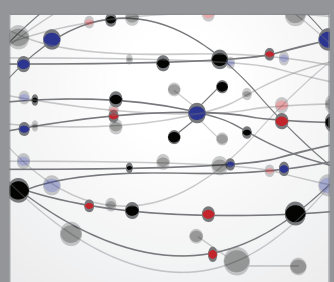

The Scientific World Journal


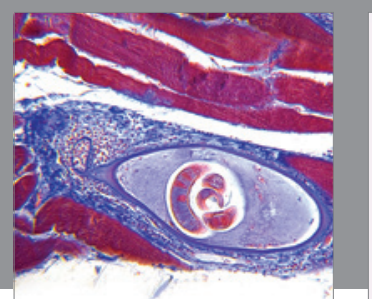

Gastroenterology Research and Practice

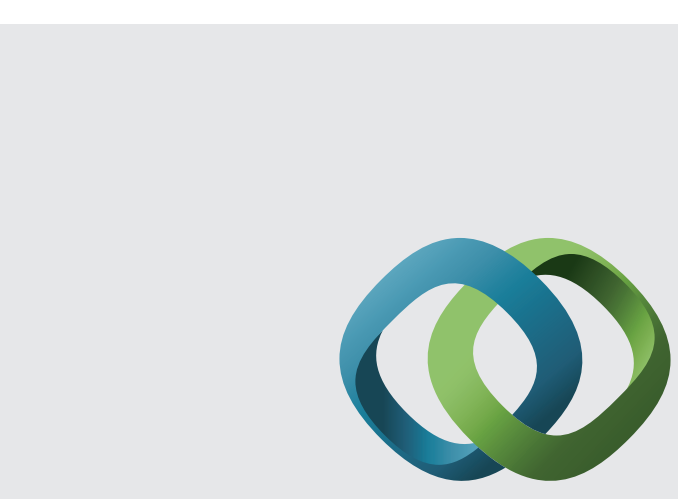

\section{Hindawi}

Submit your manuscripts at

http://www.hindawi.com
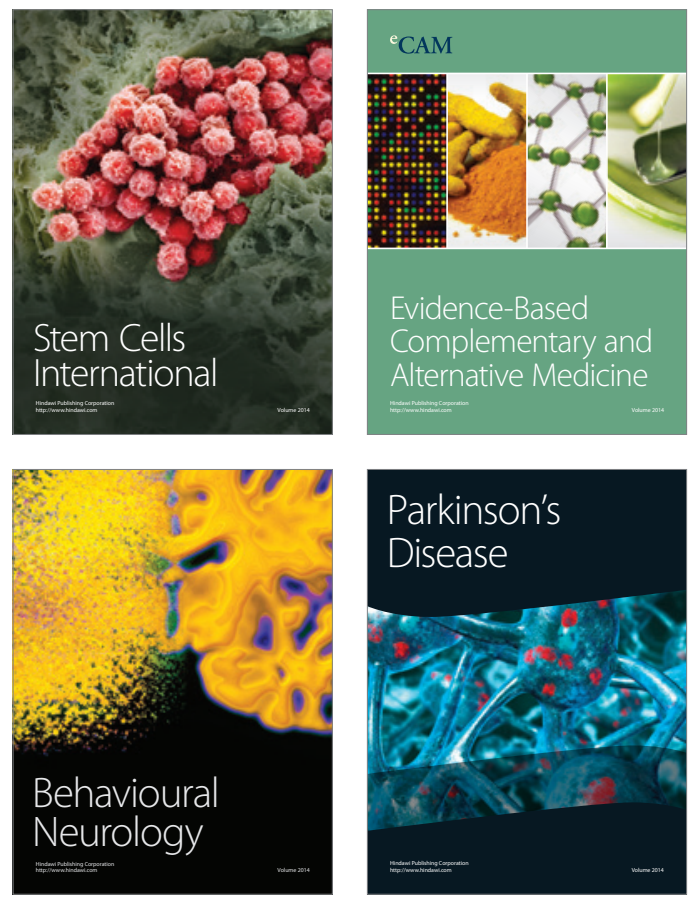


Disease Markers
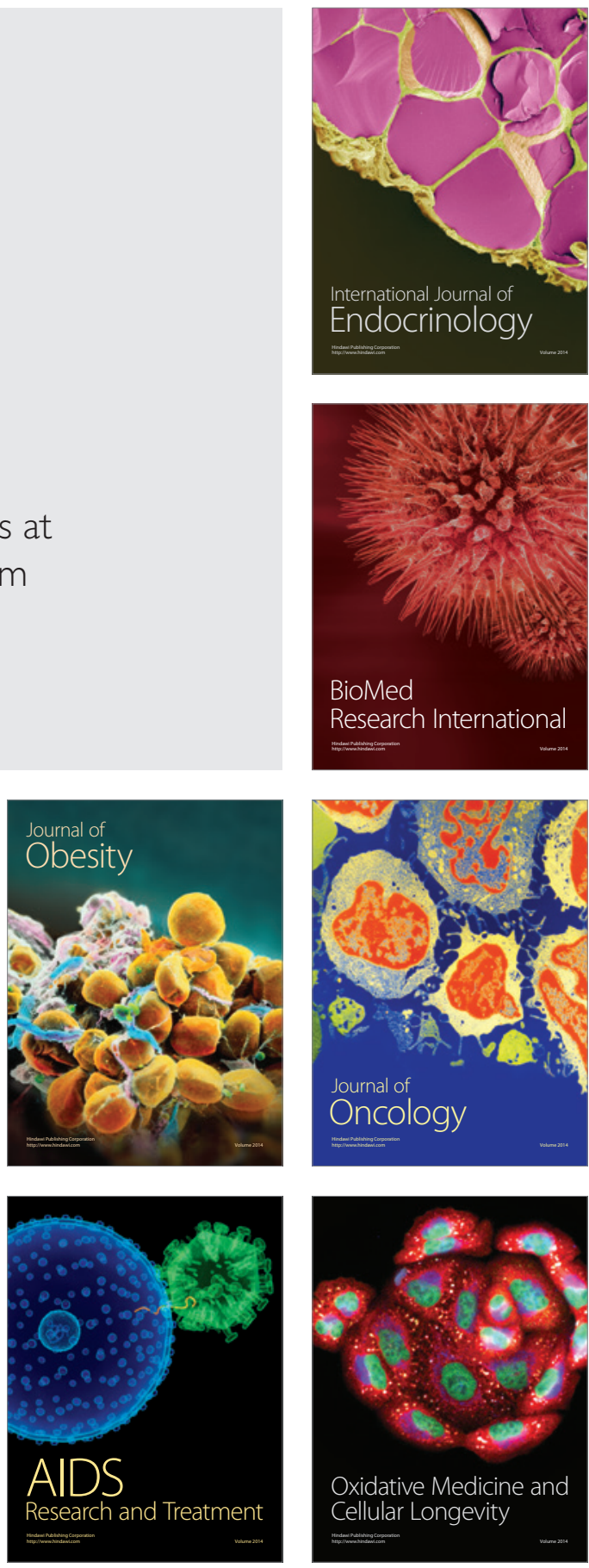